\title{
Filtragens de Recomendação de Objetos de Aprendizagem: uma revisão sistemática do CBIE
}

\author{
Walber Lins Pontes, Rômulo Martins França, Ana Paula Metz Costa, Patrícia \\ Behar
}

Universidade Federal do Rio Grande do Sul - Av. Paulo Gama, 110 - prédio 12105 - $3^{\circ}$ andar, Porto Alegre, RS, Brasil

walberpontes@gmail.com,romulomf@gmail.com, anapaulametz@gmail.com, patricia.behar@ufrgs.br

\begin{abstract}
Learning Objects have become an important didactic teaching tool in educational environments. The use of Recommender Systems to indicate these objects from some types of filtering recommendation is a difficult task assist students and teachers in search of a discipline materials. The objective is to review the literature on filtering recommendation of Learning Objects in CBIE 2008 to 2013 in order to collaborate with an overview of the area, presenting a Brazilian study on scientific production. The results are presented as the number of publications, the most cited authors, the most widely used sources and publications by authors.
\end{abstract}

Resumo: Os Objetos de Aprendizagem (OA) tornaram-se um importante recurso didático-pedagógico em ambientes educacionais. A utilização dos Sistemas de Recomendação para indicar estes objetos, a partir de alguns tipos de filtragem de recomendação, auxiliam uma tarefa árdua dos estudantes e professores na busca por materiais de uma disciplina. $O$ objetivo do trabalho é revisar as publicações sobre as filtragens de recomendação de OA do CBIE de 2008 a 2013 a fim de colaborar com uma visão geral da área, ao apresentar um estudo brasileiro sobre as produções científicas. Os resultados são apresentados a partir do número de publicações, os autores mais citados, as fontes mais utilizadas e as publicações por autores.

\section{Introdução}

Diante de uma sociedade em constante transformação, as Tecnologias da Informação e Comunicação (TIC) apresentam-se como instrumentos mediadores da aprendizagem. Os Objetos de Aprendizagem (OA) com uso dos repositórios digitais tornaram-se um importante recurso didático-pedagógico em muitos ambientes educacionais. Apesar da existência de vários repositórios digitais de OA, eles retornam muitos dados e geram uma tarefa árdua para os estudantes e professores na seleção por OA relevantes perante as suas necessidades.

Uma forma de auxiliar este processo é a utilização dos Sistemas de Recomendação para indicar os conteúdos educacionais, a partir de alguns tipos de filtragem de recomendação como o perfil do aprendiz, conteúdos dos materiais disponíveis nos ambiente virtuais ou repositórios, palavras-chaves, grupos afins, entre outros.

O trabalho justifica-se por contribuir com a área de Sistemas de Recomendação ao apresentar as produções científicas sobre as filtragens de recomendação de objetos de aprendizagem publicadas no principal evento brasileiro de Informática na Educação. 
Assim, o objetivo deste trabalho é revisar sistematicamente os trabalhos sobre as filtragens de recomendação de Objetos de Aprendizagens publicados nos anais do Congresso Brasileiro de Informática na Educação no período de 2008 à 2013 a fim de contribuir com uma visão geral do área.

A estrutura do trabalho segue com o referencial teórico sobre Sistemas de Recomendação na seção 2, os Tipos de Filtragens de Recomendação na seção 3 e as diferentes definições sobre Objetos de Aprendizagens na seção 4. Na seção 5 é exposta a metodologia do trabalho com os passos de sua execução. Na seção 6 são apresentados os dados analisados e os resultados através de gráficos. Por fim, na seção 7 é a conclusão do trabalho relacionando ao objetivo proposto.

\section{Sistemas de Recomendação}

Os Sistemas de Recomendação - SR são utilizados para identificar usuários, armazenar suas preferências e recomendar itens que podem ser produtos, serviços e/ou conteúdos, de acordo com suas necessidades e interesses. Eles surgem da dificuldade dos usuários ao encontrar os itens ou recursos que necessitam diante da grande quantidade de informações disponíveis.

De acordo com Costa et al. (2013), os Sistemas de Recomendação servem na área educacional, por exemplo, para filtrar recursos educacionais contemplando os perfis individuais dos alunos ou até mesmo perfis de um grupo de alunos de uma disciplina. Dessa forma, esses sistemas podem auxiliar o professor na composição de recursos digitais para uma disciplina e no acompanhamento dos alunos, uma vez que a preocupação em identificar e tratar perfis diferenciados é parcialmente ou até mesmo completamente resolvida com a personalização provida por esses sistemas.

$\mathrm{O}$ processo de recomendação considera múltiplas alternativas para uma solução. Na busca da escolha mais adequada geralmente realiza uma escolha direta ou por meio de recomendações prévias, conforme Maes e Shardnand (1995). Assim, considera-se a necessidade de recomendar conteúdos, elementos ou informações adequando à expectativa do indivíduo (RESNICK e VARIAN, 1997).

Cazella et al. (2009) enfatiza o desafio dos sistemas de recomendação ao realizar a combinação adequada entre expectativas dos usuários e os elementos a serem recomendados. Segundo Schafer (2000), a estrutura dos sistemas de recomendação é dividida em quatro processos: identificação do usuário, coleta de informações, estratégias de recomendação e visualização das recomendações.

A primeira parte do processo consiste na identificação do usuário, sendo opcional em um sistema de recomendação. A segunda etapa é a coleta dos dados dos usuários, que são usados na recomendação, portanto, armazenados em um banco de dados. O terceiro passo é a criação de estratégias de recomendação definidas pelos analistas de sistemas e projetistas. Por fim, a visualização das informações de forma fácil geradas pelo sistema de recomendação. Para que seja possível realizar estes quatro processos, reconhece-se a filtragem como mecanismo de geração de recomendação destes tipos de sistemas.

\section{Tipos de Filtragem}

A partir da literatura levantada neste artigo são encontrados sete tipos de sistemas de filtragem: 1) filtragem colaborativa; 2) filtragem baseada em conteúdo; 3 ) filtragem demográfica; 4) filtragem baseada em conhecimento; 5) filtragem baseada em utilidade; 6) filtragem baseada em outros contextos; 7) filtragem híbrida. 
Os dois primeiros tipos de filtragem são em geral baseados nos textos de Goldberg et al. (1992) e Resnick e Variant (1997); o terceiro, no trabalho de Montaner (2003); o quarto e quinto são encontrados em Burke (2002); o sexto é abordado por Gonzalez et al. (2007) e Nunes (2009). Por fim, filtragem híbrida é formulada em decorrência dos sistemas anteriores, encontrada em Adomaviciius e Tuhilin (2005) e Burke (2002).

A Filtragem Colaborativa baseia-se em sistemas que realizam o processo de recomendação por meio do auxílio humano, decorrente da colaboração de grupos que se interessam por elementos comuns. Tem como limitação o reconhecimento do interesse e a compreensão do indivíduo colaborador sobre o objeto, bem como sobre o próprio processo de recomendação, de acordo com Goldberg et al. (1992) e Resnick e Variant (1997).

A Filtragem baseada em Conteúdo se constitui como sistemas que aplicam o reconhecimento de conteúdos que podem ter interesse comuns implícitos ou explícitos. O seu processo de dá por abordagens distintas, mas com finalidade precípua de recomendação.

Montaner et al. (2003), destaca a filtragem demográfica por se utilizar a descrição de um indivíduo para aprender o relacionamento entre um item em particular e o tipo de indivíduo que poderia vir a se interessar, considerando as descrições das pessoas para compreensão dos relacionamentos, constituindo um perfil do usuário e classificando com base em estereótipos.

A filtragem baseada em conhecimento, a recomendação dos itens é feita baseada nas inferências das preferências do usuário e suas necessidades através de conhecimento estruturado de forma funcional. (BURKE, 2002) O mesmo autor ainda aponta a filtragem baseada em utilidade, no qual a recomendação é realizada considerando a utilidade dos itens para um determinado usuário.

Gonzalez et al. (2007) e Nunes (2009) propõem também técnicas de recomendação complementares seguindo a linha proposta por Burke (2002). Essas técnicas envolvem questões psicológicas do usuário combinados as características dos produtos e serviços, a mesma intitula-se, segundo Gonzalez et al. (2007), de filtragem baseada em outros contextos. Ele utiliza questões de Inteligência Emocional como inovação para recomendar. Nunes (2009), em uma versão estendida de filtragem baseada em outros contextos, propõe a filtragem baseada em aspectos psicológicos utilizando exemplos de Gonzalez, que usa Inteligência Emocional para melhorar a recomendação de cursos.

A Filtragem Híbrida reconhece a possibilidade de unir mais de uma técnica de filtragem propiciando a utilização simultânea de duas ou mais, com a finalidade que sejam supridas as limitações de cada modalidade, segundo Adomaviciius e Tuhilin (2005) e Burke (2002).

\section{Objetos de Aprendizagem: conceituações}

O primeiro a utilizar o termo Objetos de Aprendizagem foi Wayne Hodgins em 1994, de acordo com CEDMAD (2003). Um Objeto de Aprendizagem é uma entidade auto-contida, reusável, que pode ser claramente utilizada para aprendizagem, educacão e treinamento, representando um grande avanço no pensamento da criaç̃o de material didático reutilizável. (TEODORO et al., 2008)

Wiley (1999) conceitua os OA's como recursos digitais possíveis de serem reutilizados para suporte ao ensino. Já Tarouco (2003) amplia a compreensão ao reconhecer o OA como qualquer recurso suplementar ao processo de aprendizagem, desvinculando-o da 
necessidade de ser um elemento digital. Portanto, os OAs representam qualquer entidade digital ou não digital, que pode ser utilizada, reutilizada ou referenciada durante o processo de aprendizagem aplicado a algum tipo de tecnologia (IEEE, 2002).

Os objetos de aprendizagem possuem cinco características: 1) às informações que devem estar junto ao objeto (metadados); 2) a reutilização; 3) a acessibilidade; 4) a interoperabilidade; 5) a durabilidade; em que são apresentadas como regras de padronização do desenvolvimento dos objetivos para que eles sirvam a característica de reutilização.

Em virtude da necessidade de reutilização do Objeto de Aprendizagem, da sua granularidade e de sua disponibilidade em repositórios educacionais, é necessário reconhecer as suas características e funcionalidades. Desta forma, os Sistemas de Recomendação permitem que se selecionem em um vasto conjunto de Objetos de Aprendizagem que podem ser reutilizáveis de forma efetiva.

\section{Metodologia}

Para a realização desta revisão, o passo inicial foi a definição dos parâmetros de seleção de trabalhos de acordo com os objetivos do estudo. Os critérios de inclusão elegidos foram a abordagem explícita de algum tipo de sistema de filtragem que recomendam os objetos de aprendizagem. Os tipos de filtragem foram considerados tanto quando aparecem baseados em processos já consolidados quando apresentam alguma nova proposta para o processamento das informações.

Fixados os parâmetros de seleção, os anais do CBIE - Congresso Brasileiro de Informática na Educação publicados entre os anos de 2008 a 2013 disponíveis em http://www.cbie.org.br/index.php/anaissbie, passaram a ser revisados ano a ano. Buscou-se inicialmente nos títulos, nas palavras-chaves e nos resumos dos artigos os parâmetros de seleção definidos. Esta seleção preliminar resultou na eleição de 26 publicações.

$\mathrm{Na}$ segunda etapa do processo de revisão os artigos foram analisados na íntegra e 19 artigos atenderam de maneira satisfatória aos critérios determinados. Desses trabalhos foram identificados e sistematizados os seguintes itens: título do artigo, autores, ano de publicação, tipo de filtragem abordada no trabalho e os trabalhos referenciados como suporte teórico do sistema de recomendação e de filtragem de objetos de aprendizagem.

\section{Análise e Discussão dos Dados}

Ao se realizar o levantamento das publicações do CBIE entre 2008 e 2013 podese observar que, desde o início do período pesquisado, se encontram ao todo 19 artigos que tratam de recomendação e sistemas de filtragem de objetos de aprendizagem.

Nos anais do evento houve uma constante publicação sobre o tema, totalizando um percentual de $10 \%$ dos artigos publicados em cada ano. Em 2010 existiu uma redução do número de artigos publicados, com apenas 5,3\% dos artigos abordando sistemas de filtragem de objetos de aprendizagem. A partir de 2011 existe uma elevação substancial dos artigos publicados perfazendo $15,8 \%$ das publicações, $21,1 \%$ em 2012 e $36,8 \%$ em 2013, como apresentado no Gráfico 1. 
Gráfico 1: Número de Publicações de 2008 à 2013 no CBIE

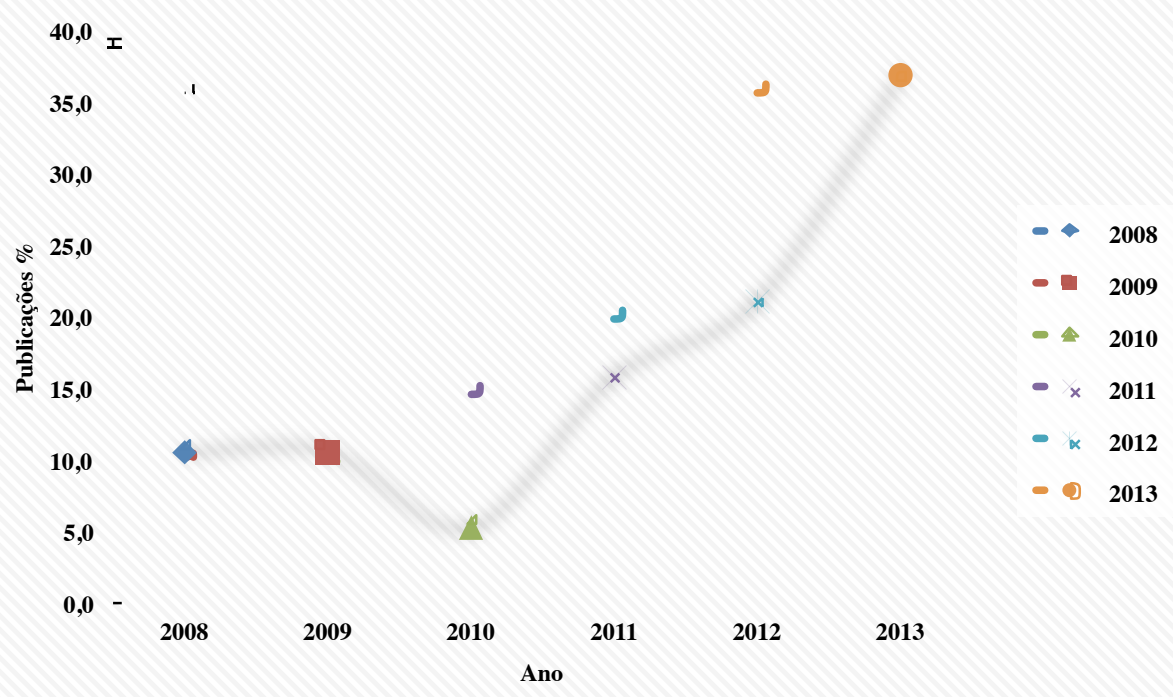

Em relação aos autores que realizaram publicações sobre os sistemas de filtragem de objetos de aprendizagem no CBIE encontram-se 54 autores distintos. Destes, 83\% realizaram apenas uma publicação, $13 \%$ realizaram 2 publicações e apenas 4\% realizaram 4 publicações, como observado no Gráfico 2.

\section{Gráfico 2: Número de Publicações por Autor}

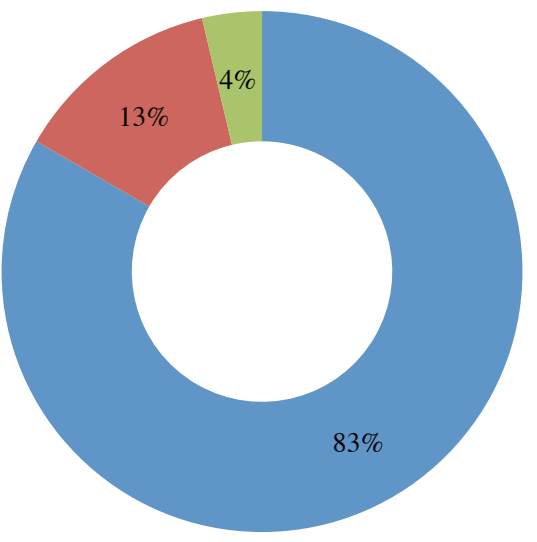

Um terceiro elemento analisado nesta revisão sistemática foram as publicações utilizadas para sustentar teoricamente os sistemas de filtragem de objetos de aprendizagem. Nos 19 artigos, que fazem parte desta revisão, são citados 45 trabalhos distintos utilizados como referencial. Desses, $89 \%$ dos artigos foram citados apenas uma única vez e $11 \%$ foram citados duas vezes nas publicações, conforme observado no Gráfico 3. 


\section{Gráfico 3: Número de Fontes Utilizadas}

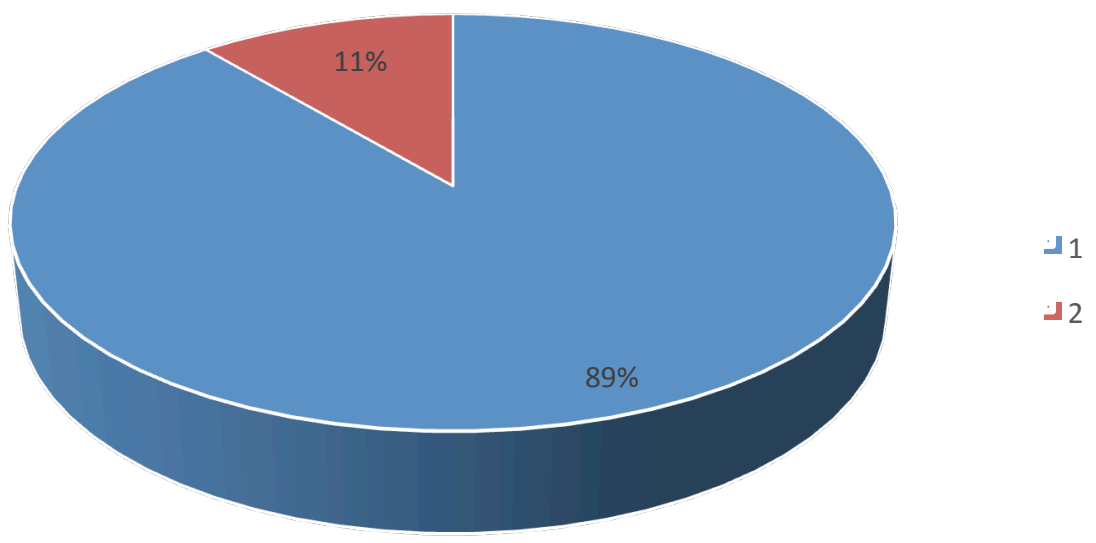

Ao se considerar individualmente os autores que suportaram as publicações no CBIE encontram-se 82 autores. Desses 70\% foram citados apenas uma única vez, 6 autores aparecem com 4 citações e apenas 1 autor foi citado 5 ou 7 vezes, como observado no Gráfico 4. Os autores que apresentam 5 ou 7 citações apresentam vínculo com o Programa de Pós-Graduação em Informática na Educação - PGIE da Universidade Federal do Rio Grande do Sul.

\section{Gráfico 4: Número de autores citados}

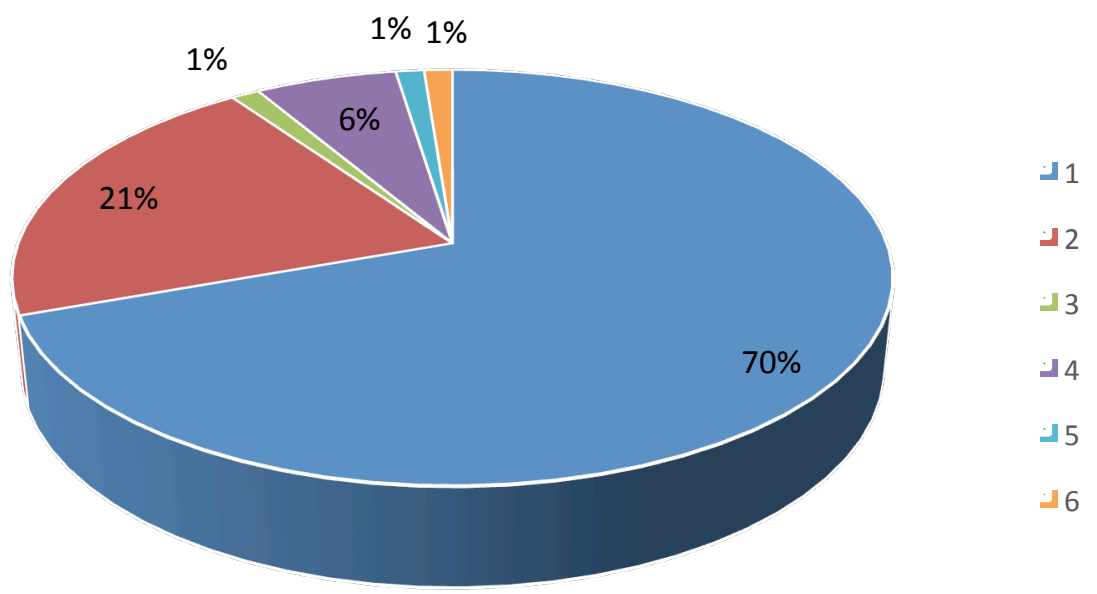

Com relação ao sistema de filtragem de objetos de aprendizagem, os 19 artigos foram categorizados segundo a Tabela 1:

\section{Tabela 1: Categorização dos artigos}

Filtragem a partir de dados inseridos em relação ao estado de desenvolvimento do aluno,

1 do conteúdo a ser ensino e Ontologias baseadas em teorias de aprendizagem

2 Filtragem a partir das dificuldades de aprendizagem registradas (performance do aluno)

3 Filtragem Híbrida: Filtragem Colaborativa e Recomendação considerando Competências 


\begin{tabular}{r|l|}
\hline 4 & Filtragem por Modelagem de Estereótipos e por Perfil de Aprendizagem \\
\hline 5 & $\begin{array}{l}\text { Propõe uma etapa de pós processamento após os processos tradicionais de filtragem } \\
\text { (Baseada em Conteúdo, Colaborativa ou Híbrida). Tal etapa consiste na comparação } \\
\text { metadados que descreve o OA }\end{array}$ \\
\hline 6 & $\begin{array}{l}\text { Filtragem Híbrida: Filtragem Colaborativa e Filtragem Baseada em Conteúdo (a partir } \\
\text { de informações do currículo Lattes dos usuários e de metadados sobre os documentos } \\
\text { digitais) }\end{array}$ \\
\hline 7 & Sistema de Recomendação Híbrido e Recomendação Sensível ao contexto \\
\hline 8 & $\begin{array}{l}\text { Recomendação Híbrida: a) recomendação não personalizada (tipo de recomendação } \\
\text { que sugere itens levando em consideração a avaliação do item, desconsiderando o } \\
\text { perfil do usuário); b) recomendação baseada no conteúdo e c) recomendação baseada } \\
\text { na filtragem colaborativa }\end{array}$ \\
\hline 9 & $\begin{array}{l}\text { Sistema de Recomendação Híbrido, considerando Filtragem Colaborativa e Filtragem } \\
\text { Baseada em Conteúdo e Filtragem Baseadas em Competências, considerando as } \\
\text { competências a serem desenvolvidas pelo aluno. }\end{array}$ \\
\hline 10 & Recomendação Baseada em Conteúdo e Recomendação Sensível ao Contexto \\
\hline 11 & Filtragem Híbrida: Baseada em Conteúdo, Colaborativa e Sensível ao Contexto \\
\hline 12 & $\begin{array}{l}\text { Filtragem Baseada em Conteúdo (Ontologia de descrição das peças e Ontologias de } \\
\text { contexto estático e dinâmico do usuário) }\end{array}$ \\
\hline 13 & $\begin{array}{l}\text { Filtragem Baseada em Conteúdo (Compara a utilização de Hashtags com a descrição } \\
\text { dos OAS) }\end{array}$ \\
\hline 14 & Filtragem Colaborativa (utilizando dados demográficos) \\
\hline 15 & Filtragem Colaborativa e técnica de agrupamento de usuários com perfis similares \\
\hline 16 & $\begin{array}{l}\text { Filtragem por Conteúdo (considerando os perfis dos aprendizes) e informações do } \\
\text { Contexto }\end{array}$ \\
\hline 17 & Filtragem Baseada em Conteúdo (considerando o Estilo de Aprendizagem do aluno) \\
\hline 18 & Filtragem baseada em similaridade de sessões \\
\hline 19 & Filtragem baseada em contexto utilizando Algoritmo Genético \\
\hline
\end{tabular}

É possível observar que parte das recomendações se utiliza da filtragem por conteúdo, podendo utilizar-se de outros sistemas para ampliar as avaliações para a recomendação. Reconhece-se também a relevância dos sistemas de colaboração, uma vez que sete dos dezenove artigos pesquisados se utilizam desta estrutura de filtragem.

Observa-se ainda que, com a aplicação de uma estrutura híbrida neste contexto, a totalidade das recomendações considera uma estrutura demográfica ou de conhecimento como sistema de filtragem para adequar o conteúdo às características individuais dos usuários.

\section{Conclusão}

Realizou-se uma revisão sistemática sobre as filtragens de recomendação de objetos de aprendizagem publicados nos anais do Congresso Brasileiro de Informática na Educação no período de 2008 a 2013.

A partir da análise e dos dados apresentados verificou-se um crescimento nas produções científicas publicadas, no referido congresso sobre o tema pesquisado, a partir do ano de 2011 com aumento significativo que ultrapassa os $30 \%$. 
Um ponto importante que merece atenção é quanto aos tipos de filtragem e como eles utilizam determinados dados para contribuir no contexto da recomendação como dados demográficos, perfis de usuários, agrupamentos de usuários, estilos de aprendizagens dos alunos, colaboração, estereótipos dos alunos, dados dos currículos lattes, entre outros.

Informações importantes podem ser citadas além do número de publicações como: autores mais citados, fontes mais utilizadas e publicações por autores. Enfim, esta pesquisa serve de base para o desenvolvimento da área de sistemas de recomendação de objetos de aprendizagem apresentando uma visão temporal das produções no principal congresso do contexto brasileiro.

Como trabalhos futuros poderá ser realizada uma revisão sistemática sobre sistemas de recomendação de objetos de aprendizagem em algumas bases científicas internacionais. Outro viés é relacionar os termos aqui pesquisados com as competências voltadas para educação, por ser uma temática relevante no cenário atual diante de requisitos que tornam viáveis a prática de ensino e aprendizagem através das novas tecnologias da informação e da comunicação.

\section{Referências}

Adomavicius, G.; Tuzhilin, A. (2005). Toward the Next Generation of Recommender Systems: ASurvey of the State-of-the-Art and Possible Extensions. IEEE Transactions on Knowledgeand Data Engineering, New York, v. 17, n. 6p. 734-749.

Botelho, Carlos Alberto; pimentel, Edson P.; senger, Hermes; stiubener, Itana. Personalização em Sistemas de Gerenciamento da Aprendizagem em Conformidade com o Padrão SCORM. In: Anais do Simpósio Brasileiro de Informática na Educação. 2009.

Burke, R. (2002). Hybrid recommender systems: Survey and experiments. User Modeling and User-Adapted Interaction, Dordrecht, v. 12, p. 4, p. 331-370.

Casagrande, Marcus Filipi Rosso; kozima, Gustavo; willrich, Roberto. Técnica de Recomendação Baseada em Metadados para Repositórios Digitais Voltados ao Ensino. In: Anais do Simpósio Brasileiro de Informática na Educação. 2013.

Cazella, S. C., Bhear, Patricia, schneider, Daisy Schneider, SILVA, Ketia Kellen da, freitas, Rodrigo. Desenvolvendo um Sistema de Recomendação de OAs baseado em Competências para a Educação: relato de experiências. Anais do $23^{\circ}$ Simpósio Brasileiro de Informática na Educação (SBIE 2012) Rio de Janeiro, 26-30 de Novembro de 2012

Cazella, S. C.; Reategui, E.; Machado, M.; Barbosa, J. (2009) Recomendação de OAs Empregando Filtragem Colaborativa e Competências. In: Simpósio Brasileiro de Informática na Educação (SBIE).

Costa, E.; Aguiar, J.; Magalhães, J.; Sistemas de Recomendação de Recursos Educacionais: conceitos, técnicas e aplicações. Cap. 03, II Congresso Brasileiro de Informática na Educação, 2013.

Moura, Íthalo Bruno Grigório de; lima, João de Deus; Mendes Neto Francisco Milton; maia, Paulo Sérgio Sousa. Musert: Um Museu Virtual em 3D com Recomendação 
Personalizada de Conteúdo. In: Anais do Simpósio Brasileiro de Informática na Educação. 2012.

Dorça, Fabiano A.; Silva, Daydson H.; lima, Luciano V.; Fernandes, Márcia A.; Lopes, Carlos R. Uma Abordagem para Geração Automática de Conteúdo Personalizado Através da Recomendação Estocástica de Objetos de Aprendizagem no Processo de Ensino em Sistemas Adaptativos e Inteligentes para Educação. In: Anais do Simpósio Brasileiro de Informática na Educação. 2013.

Ferreira, Luís Gustavo Araujo; Barbosa, Jorge Luis Victória; Gluz, João Carlos. Um Modelo de Recomendação Ubíqua de Conteúdo para Grupos de Aprendizes. In: Anais do Simpósio Brasileiro de Informática na Educação. 2013.

Ferreira, Luis Gustavo Araujo; Gluz, João Carlos; Barbosa, Jorge Luis Victória. Um Modelo Multiagente para Recomendação de Conteúdo Educacional em um Ambiente Ubíquo. In: Anais do Simpósio Brasileiro de Informática na Educação. 2012.

Ferro, Márcio Robério da Costa; Nascimento Júnior, Hélio Martins; Paraguaçu, Fábio; Costa, Evandro de Barros; Monteiro, Larissa Artemis Luna. Um modelo de sistema de recomendação de materiais didáticos para ambientes virtuais de aprendizagem. In: Anais do Simpósio Brasileiro de Informática na Educação. 2011.

Goldberg, D., Nichols, D., Oki, B. M., Terry, D. (1992). Using collaborative filtering to weave an information Tapestry. Communications of the ACM, New York, v.35, n.12, p. 61-70, Dec.

Gonzalez, G., De La Rosa, J.L., and Montaner, M. (2007). Embedding Emotional Context in Recommender Systems. In The 20th International Florida Artificial Intelligence Research Society Conference-FLAIRS, Key West, Florida.

Gotardo, Reginaldo; Cereda, Paulo Roberto Massa; Hruschka Junior, Estevam Rafael. Predição do Desempenho do Aluno usando Sistemas de Recomendação e Acoplamento de Classificadores. In: Anais do Simpósio Brasileiro de Informática na Educação. 2013.

IEEE. IEEE Learning Technology Standards Committee. Draft Standard for Learning Object Metadata (IEEE 1484.12.1-2002). 2002, 44p.

Isotani, Seiji; Isotani, Naoko; Isotani, Sadao. Ontologias e Web Semântica no Suporte ao Ensino Colaborativo em Salas de Aula Presenciais. In: Anais do Simpósio Brasileiro de Informática na Educação. 2008. p. 521-531.

Silva Júnior, Luiz Cláudio Ferreira da; HERNÁNDEZ-DOMÍNGUES, Arturo. Um Sistema Tutor Móvel no contexto de um Framework de Sistemas de Ensino On-line. In: Anais do Simpósio Brasileiro de Informática na Educação. 2008. p. 696-705.

Jácome Júnior, Luiz; Mendes Neto, Francisco Milton; DA Silva, Luiz Cláudio Nogueira. Uma Abordagem Baseada em Algoritmo Genético para Recomendação de Objetos de Aprendizagem Sensível ao Contexto do Estudante. In: Anais do Simpósio Brasileiro de Informática na Educação. 2012. 
Maes, P.; Shardanand, U. (1995). "Social information filtering: Algorithms for automating "word of mouth", In: Human Factors in Computing Systems. Proceedings..., 1995, p. 210-217.

Martins Nj, Hélio; Costa, Evandro B.; Oliveira, Thyago T. M.; Silva, Alan P.; Bittencourt Ig I. Sistema de Recomendação Híbrido para Bibliotecas Digitais que Suportam o Protocolo OAI-PMH. In: Anais do Simpósio Brasileiro de Informática na Educação. 2011.

Montaner, M., López, B., De la Rosa, J.L. (2003). A Taxonomy of Recommender Agents on the Internet. Artificial Intelligence Review. Netherlands : Kluwer Academic Publishers, pp. 285-330, Aug.

Muniz, Raphael de Carvalho; Souzam Rafael Castro de; Mendes Neto, Francisco Milton; Burlamaqui, Aquiles Medeiros Filgueira. Um Sistema Multiagente para Recomendação de Conteúdos Educacionais para TV Digital Interativa. In: Anais do Simpósio Brasileiro de Informática na Educação. 2013.

RESNICK, P. e VARIANT, H. R. (1997) Recomender systems. Comunications of the ACM, New York, V. 40, n. 3, pp. 55-58, mar.

Nunes, M. A. S. N. (2009). Recommender Systems based on Personality Traits:Could human psychological aspects influence the computer decision-making process?. 1. ed. Berlin: VDM Verlag Dr. Müller. v.1. 140 p.

Primo, Tiago Thompsen; Vicari, Rosa Maria; DA Silva, Julia Marques Carvalho. Rumo ao uso de metadados educacionais em sistemas de recomendação. In: Anais do Simpósio Brasileiro de Informática na Educação. 2010.

Silva, Luiz Cláudio Nogueira da; Mendes Neto, Francisco Milton; Jácome Júnior, Luiz. Mobile: Um ambiente multiagente de aprendizagem movel para apoiar a recomendacao sensivel ao contexto de objetos de aprendizagem. In: Anais do Simpósio Brasileiro de Informática na Educação. 2011.

Tarouco, Liane; Fabre, Marie-Christine Julie Mascarenhas; Tamusiunas, Fabrício Raupp. Reusabilidade de objetos educacionais. Revista Novas Tecnologias na Educação. Porto Alegre, p. 1-11. 2003.

Teodoro, G.; Carvalho, M. B.; Comassetto, L. S. Compartilhamento e Reusabilidade de Objetos de Aprendizagem. In: V Congresso Bras. de Ensino Superior a Distância, 2008, pp. 1-10.

Wiedemann, Tiago; Barbosa, Jorge LV; Rigo, Sandro J. Um Modelo para Recomendação de Objetos de Aprendizagem Baseado em Similaridade de Sessões. In: Anais do Simpósio Brasileiro de Informática na Educação. 2013.

Wiley, D. A. Learning objects and the new CAI: So what do I do with a learning object? 1999. Disponível em: $<$ http://penta3.ufrgs.br/objetosaprendizagem/11wiley_traducao.doc $>$. Acesso em: 02 de março de 2014. 